\title{
Effect of psychological intervention on post-traumatic stress symptoms and pregnancy outcomes among women with previous recurrent abortion
}

\author{
Hanaa Mohamed Abo Shereda ${ }^{1}$, Amera Bekhatro Awad Allah Rashed ${ }^{* 2}$, Eman Shokr ${ }^{3}$ \\ ${ }^{1}$ Psychiatric Mental Health Nursing Department, Faculty of Nursing, Menoufia University, Egypt \\ ${ }^{2}$ Maternal and Newborn Health Nursing Department, Faculty of Nursing, Menoufia University, Egypt \\ ${ }^{3}$ Community Health Nursing Department, Faculty of Nursing, Menoufia University, Egypt
}

Received: July 30, 2018

Accepted: September 24, $2018 \quad$ Online Published: September 30, 2018

DOI: $10.5430 /$ jnep.v8n12p123

URL: https://doi.org/10.5430/jnep.v8n12p123

\begin{abstract}
Background: Recurrent abortion is both physically and psychologically a traumatic experience. Some women also experience anxiety, anger, posttraumatic stress, and guilt about future childbearing. Supportive psychotherapy aims to minimize deterioration and maximize competence within the limitations imposed by the illness. The purpose of the study was to study the effect of psychological intervention on post-traumatic stress symptoms and pregnancy outcomes among pregnant women with previous recurrent abortion.

Methods: Research design: a quasi-experimental research design (with pre-post measurements) was used in this study. Subjects: A purposive sample of 40 pregnant women was recruited. Tools: Two tools were used for data collection: interviewing questionnaire and posttraumatic stress disorder checklist.

Results: There was a statistically significant difference between pre and post intervention results regarding post traumatic stress symptoms severity after the intervention. Psychological intervention was associated with positive pregnancy outcomes.

Conclusions and recommendations: The first research hypothesis was accepted as it was found that the psychological intervention decreased post traumatic stress symptoms among studied women. The second hypothesis was also accepted as the psychological intervention was associated with positive pregnancy outcomes. Manage women with recurrent abortion within the purview of holistic nursing practice which address the bio-psychosocial responses of abortion.
\end{abstract}

Key Words: Psychological intervention, Post-traumatic stress symptoms, Pregnancy outcomes, Recurrent abortion

\section{INTRODUCTION}

Abortion is a common condition that happens between $12 \%$ and $24 \%$ of confirmed pregnancies. ${ }^{[1]}$ Recurrent abortion can be defined as: three or more consecutive spontaneous abortions. ${ }^{[2]}$ Although this definition has changed for women who have had two consecutive pregnancy losses in cases where fe- tal heart activity had been detected before pregnancy loss. ${ }^{[3]}$ Recurrent abortion represents about $1 \%$ of spontaneous abortions. ${ }^{[4]}$ The cause of recurrent abortion is often unknown or idiopathic although many factors can play role as genetic, immunologic, anatomic abnormalities, endocrinopathies and infections. $^{[2]}$

\footnotetext{
* Correspondence: Amera Bekhatro Awad Allah Rashed; Email: amera.rashed@yahoo.com; Address: Maternal and Newborn Health Nursing Department, Faculty of Nursing, Menoufia University, Egypt.
} 
However the experience of abortion may be relatively common, the feelings of isolation and blame felt by women who have had abortions are often poorly appreciated by not only their immediate support system, but also by health professionals too. ${ }^{[4]}$

Recurrent abortion is psychologically more painful than firsttime abortion. It seems to have a complicated effect on a woman's self esteem, perception of herself as successful and her relationship with significant others. The desire of the pregnancy, number of live children, and the available support are the most important variables that determine the effect of pregnancy loss. Recurrent abortion leaves a cumulative effect, which can appear in many aspects of a woman's functions. ${ }^{[4]}$

Amongst the main psychiatric syndromes, both depression and anxiety are the commonest. In a recent study, $32 \%$ of the sample was classified as clinically depressed by self-report questionnaire. Between $43 \%$ and $70 \%$ of the same study sample reported biological symptoms of depression, as loss of sleep, weight and also appetite. ${ }^{[5]}$

Post-traumatic stress (PTS) can be defined as an anxiety disorder with the following cluster of symptoms: reexperiencing (nightmares and flashbacks), persistent avoidance of reminders (loss of memory of the event) and hyperarousal (irritability, difficulty concentrating) ${ }^{[6]}$ Post abortive post-traumatic stress disorder (PTSD) is a well known term. It has been acknowledged in the Diagnostic and Statistical Manual of the American Psychiatric Association-fourth edition-(DSM-IV) since 1994.

A gap exists within healthcare personnel between identifying and treating post abortive PTSD however 30\% of women worldwide experience such condition. Younger women are at the highest risk for that problem after abortion. Limited data for accurate and effective interventions to relieve psychological distress after abortion were found.

Many authors highlighted the importance of psychological support following recurrent abortion. There are two studies evaluated the effect of a specialized clinical approach. ${ }^{[7,8]}$ Swanson et al. ${ }^{[5]}$ evaluated the effect of a specific, brief therapeutic approach composed of three sessions providing women who have had abortions with the opportunity to discuss their experience and the difficulties they were facing. Women who received this intervention showed early in evaluations that they had lower scores of post traumatic stress symptoms. Several studies, however, reported no significant effect for a structured therapeutic approach using a midwife. ${ }^{[9]}$ Another author studied the impact of a one-session psychological debriefing; there were no significant results four months following this study while measuring post traumatic stress symptoms. ${ }^{[10]}$ Another study showed promising results using data from previous studies to develop a brief telephone support intervention. ${ }^{[11]}$

\subsection{Operational definitions}

Psychological Intervention: Is a wide array of behavioral and psychotherapeutic treatments designed to reduce psychological distress and maladaptive behavior and to increase adaptive behavior, typically through counseling, support, interaction, or instruction. In the current study, the psychological intervention was carried out in the form of a program with 6 sessions and a post-intervention measurement of symptoms severity.

Post-Traumatic Stress Symptoms: A cluster of symptoms triggered by exposure to a traumatic event (recurrent abortion) that threatened the physical integrity of self. After exposure, the patient relives the traumatic event, with persistent avoidance of situations associated with the trauma and hyperarousal. It was measured using posttraumatic stress disorder checklist.

Pregnancy Outcomes: Results of pregnancy, such as occurrence of pregnancy complications, congenital malformations, lower birth weight, mode of delivery and preterm labor or stillbirth.

Recurrent Abortion: According to the WHO, it is at least three or more consecutive pregnancy losses before the 22nd gestational week. In the current study it means 2-5 times of pregnancy loss.

\subsection{Significance of the study}

Women in developing countries experience psychological consequences of a miscarriage at rates that are comparable with or higher than those in developed countries. In Egypt, the prevalence of PTSS after recurrent abortion was observed to be $30 \%$ one month after the loss and $10 \%$ four months post loss. ${ }^{[12]}$ In particular, the contribution of recurrent abortion to women emotional well-being and their pregnancy outcomes is still not clearly understood.

Women who experience recurrent abortion and go through new pregnancy need supportive psychological intervention to maintain psychological integrity and in turn achieve positive pregnancy outcomes.

By reviewing available literature; many non-therapeutic approaches for managing PTSS and improving pregnancy outcomes were evaluated by different studies. ${ }^{[1]}$ Unfortunately, many showed no marked effects on reducing post traumatic stress symptoms or improving pregnancy outcomes. Thus, simply discussing the experience of PTSS following recur- 
rent abortion doesn't seem to have any notable positive impact on wellbeing. The reality is that providing long-term psychological therapy or, at the very least, several sessions, can be expensive, and is not usually accepted by women but it is considered the most exclusive nursing management strategy for PTSS. ${ }^{[13]}$

Based on the previously mentioned literature; there is a gap of knowledge in this research point in terms that the effective intervention was not yet studied. So, the researchers try conducting psychological intervention for recurrent abortion in order to help women overcome PTSS and improve their pregnancy outcomes.

\subsection{Purpose of the study}

The aim of the study was to assess the effect of psychological intervention on post-traumatic stress symptoms and pregnancy outcomes among women with previous recurrent abortion.

\subsection{Research hypotheses}

The psychological intervention will decrease post-traumatic stress symptoms severity among women with previous recurrent abortion.

The psychological intervention will improve pregnancy outcomes among women with previous recurrent abortion.

\section{Methods}

\subsection{Research design}

A quasi experimental design was used in conducting this study with pre-post intervention measurement of PTSS severity. Such design fits the nature of the problem under investigation. This type of research design involves one or more group of subjects observed before and after implementation of an intervention. ${ }^{[14]}$

\subsection{Setting}

The study was conducted at two governmental maternal and child health centers $(\mathrm{MCH})$ at Shebin El Koom (Qebly and Bahary), Menoufia Governorate, Egypt. These centers were purposively selected because they are known to present services to large sector of the governorate population thus having a high flow rate of pregnant women.

\subsection{Subject}

The study sample comprised of 47 pregnant women but only 40 completed the study (the other 7 were dropped out due to abortion and discontinuation of session attendance). They were recruited purposively according to the following inclusion criteria: with previous recurrent spontaneous abortion ( 2 to 5 abortions), currently pregnant, 1 to 4 months after the last previous abortion, suffering from post traumatic stress symptoms as a result of repeated abortions and free from any other psychiatric disorder. Exclusion criteria were: recurrent abortion more than 5 times, women who are receiving any other psychotherapy during the study period and women taking any psychiatric drugs.

The sample size was determined through power analysis as follows:

Based on post-intervention values of psychological intervention reported in Swanson's study, ${ }^{[5]} 40.34 \pm 1.20$ was the reported size of intervention group. The sample size was calculated to be 40 pregnant women, considering $\mathrm{CI}=95 \%$ and power $=90 \%$ (15\% dropout $)$.

The filed work: The data collection was conducted from March 2017 to April 2018.

\subsection{Maneuver of intervention}

The current study was carried out in four consecutive phases: preparatory, intervention framing, implementation and evaluation phases.

Preparatory Phase: An extensive reviewing of electronic data related to pregnancy, recurrent abortion, post traumatic stress and psychological impact of previous abortion on current pregnancy outcomes was done. A review of literature to collect relevant knowledge pertinent to study was also used in developing data collection instruments.

Intervention Framing Phase: The psychological intervention was designed by the researchers and validated by a panel. The intervention was in the form of 6 sessions. The sessions' contents were documented in a written form to be available for both researchers. A guiding booklet and an audio CD detailing the psychological intervention were prepared by the researchers and evaluated by the same panel.

Implementation Phase: The researchers purposively selected eligible women; 20 from MCH Qebli and 20 from $\mathrm{MCH}$ Bahary. Then the total number of women were divided into 8 groups (each contain 5 women) for facilitating communication and session attendance.

Participants received all sessions at the $\mathrm{MCH}$ centers and each lasted for 60 minutes. Sessions were given along 6 weeks at Sunday or Monday from each week and by rotation between the two study settings.

Session 1 was devoted to explaining the purpose of the study, its method also obtaining an oral informed consent, sitting time for other sessions and identifying methods of contacting with the researchers. It involved also a base line evaluation of (a) women main data listed in tool I, (b) post-traumatic 
stress symptoms severity using tool II.

The intended learning outcomes of the psycho-educational program sessions were:

Knowledge and understanding: List the importance of having psychological status integrity to go through pregnancy safely; Recall the complications of post-traumatic stress symptoms on fetal and maternal general condition.

Intellectual skills: Correlate the relationship between post traumatic stress symptoms and recurrent abortion; Summarize the role of the psycho-educational program in decreasing post-traumatic stress symptoms and improving pregnancy outcomes.

General and transferable skills: Value the importance of psychological intervention in decreasing post-traumatic stress symptoms and improving pregnancy outcomes; Use adaptive coping strategies in dealing with PTSS for improving pregnancy outcomes.

Attitude: Communicate effectively with the researchers to control symptoms severity.

Teaching Materials: PowerPoint presentation using lab top computer, images and videos.

Session 2: Orientation and Introduction

Session 2 goal: To build trust, respect, and emotional safety among participants.

\section{Session 2 contents:}

(1) Introduction about pregnancy and the importance of being in a state of good psychological wellbeing for better outcomes.

(2) Introduction about the program.

(3) Describe purpose, origins, early phase the intervention.

(4) Inform that this is an initial intervention.

(5) Emphasize pre-test, posttest, as part of program completion.

(6) Encourage participants to inform providers of worsening of symptoms.

(7) Encourage participants to identify own goals for intervention.

(8) State expectation.

The booklet and the audio CD were presented to women at the end of this session.

Session 3: Psycho education of nature of post abortion stress symptoms

Session 3 goal: To increase understanding and acceptance of post abortion psychological problems.
Session 3 rationale: Increasing awareness, identification, and understanding of post abortion psychological problems can enhance self-acceptance.

Session 3 objectives:

(1) Participants will recognize post-traumatic stress as response to a abortion.

(2) Participants will identify own symptoms of postraumatic stress.

(3) Participants will reduce anxiety by normalizing symptoms of pos-traumatic stress.

\section{Session 3 contents:}

(1) Provide education for symptoms of psychological stress responses.

(2) Symptoms of post abortion distress.

(3) Identify target symptoms for intervention.

(4) Risk factors for post abortion distress.

Session 4: Psychotherapeutic processing of post-traumatic stress symptoms

Session 4 goal: To reduce the most disturbing intrusive and avoidant symptoms of distress by an initial processing of the pregnancy and abortion experience.

Session 4 rationale: By processing key symptoms of distress associated with the pregnancy/abortion experience (emotions of anger, guilt, sadness, fear, etc.) and cognitions (conflicts, condemnation etc.), participants will reduce anxiety. Unresolved or avoided psychological states can increase anxiety.

\section{Session 4 objectives:}

(1) Participants will reduce the intrusive symptoms of distress by limited dosing of disclosure of pregnancy and abortion experience.

(2) Participants will reduce the avoidant symptoms of distress by limited dosing of disclosure of pregnancy and abortion experience.

(3) Participants will exchange positive support, validation, and identification among group members.

\section{Session 4 contents}

(1) Identification of intrusive symptoms of PTSD.

(2) Clarification of the avoidant symptoms of PTSD.

(3) Allow sharing of positive support, validation of post abortion symptoms and changing of experiences.

Session 5: Skill-Building Coping Module

Session 5 goal: To increase positive coping behaviors, introduce new skills, and increase support resources, in order to improve functional progress. Reduce avoidant behaviors, maladaptive or risk-taking behavior. 
Session 5 rationale: Avoidance of distress results in maladaptive coping. Maladaptive coping can result in dysfunctional grieving, substance abuse, and self-destructive behaviors. Maladaptive coping results in increased mental health problems and decreases functioning after abortion.

\section{Session 5 objectives:}

(1) Participants will identify triggers that activate symptoms of post abortion distress.

(2) Participants will identify maladaptive coping behaviors used to manage symptoms. Participant will describe steps to replace these with positive coping skills. Participants will recognize limits, and areas that require further attention for eventual mastery of distress.

Session 5 contents: Apply coping skills to competently manage distress.

Session 6: Spiritual Support Processing Module

Session 6 goals:

(1) To reduce spiritual distress associated with abortion.

(2) To offer opportunity for closure, containment, giving meaning, and hope for renewed future.

Session 6 rationale: Some report dramatic relief of symptoms with mourning and goodbye rituals after abortion. By increasing awareness, responsibility, and accountability, participants will increase self-efficacy and sense of personal control.

\section{Session 6 objectives:}

(1) Participant will verbalize optimism/hope for future. (Renewal).

(2) Participant will demonstrate tasks toward closure of grieving such as saying goodbye, receiving forgiveness, reframing circumstance toward acceptance of self and others. (Re-frame).

(3) Participant will identify specific and modifiable behaviors that contributed to unintended pregnancy or abortion. Participant will describe plan of increased protection, responsibility, and competence for future. (Resolve).

\section{Session 6 contents:}

(1) Way of closing their experience and developing new future plans.

(2) Developing new strategies around reminders, trigger that will arise surrounding their pregnancy/abortion experience.

(3) Methods of present and future orientation with realistic hope.
(4) Reinforcement of what has been gained throughout the intervention.

Evaluation Phase: The researchers followed up the participants at:

- The end of the sixth session by evaluating the posttraumatic stress symptoms severity (tool II).

- Along the course of pregnancy for any complications (tool I).

- During and immediately after delivery for evaluating pregnancy outcomes (tool I).

\subsection{Instruments}

Two tools were used during the course of this study: (I) interviewing questionnaire and (II) post-traumatic stress symptoms checklist.

I-The interviewing questionnaire: It was developed by the researcher and submitted to validity and reliability tests. It was used to evaluate sociodemographic data, obstetric and gynecological history and pregnancy outcomes it included: (A) Basic data of studied women, age, duration of marriage, education, occupation, residence and income... (B) Number of abortions and gestational age of current pregnancy. (C) Pregnancy outcomes. Validity of the interviewing questionnaire was ascertained through an expert panel (medical and nursing staff) to assure content validity. They were asked also to judge the items for completeness and clarity. Suggestions were incorporated into the tool. Then test-retest reliability was applied. The tool proved to be strongly reliable.

II-Post-Traumatic Stress Disorder Checklist: It was adapted from "posttraumatic stress disorder check list" (PCL) developed by Weathers et al. ${ }^{[15]}$ and translated into Arabic by the researchers. It was used to assess post traumatic symptoms severity before the intervention and after the 6th session. The checklist is a widely used instrument that measures distress related to PTS symptoms. It consists of 17 items that correspond to PTSD Diagnostic and Statistical Manual of the American Psychiatric Association IV diagnostic criteria (DSM-IV). Women were asked to rate how much they were bothered by the repeated abortion on a 5-point scale (ranging from 1 "not at all" to 5 "extremely"), and totals range from 17 to 85 with higher scores indicating greater symptom severity.

Only women who met all of the DSM-IV criteria from A to F were classified as having a diagnosis of PTSD. Ongoing PTSD was measured with an additional question asking "if the trauma symptoms are present right now". Different terms have been used to denote individuals who only partly meet the diagnostic criteria of PTSD: sub-threshold PTSD, partial PTSD, or posttraumatic stress symptoms (PTSS). In the 
present study, the term PTSS was used and was defined as prevalence of A1 and A2 criteria together with one or more of the re-experiencing, avoidance or hyper arousal symptoms (B-C-D criteria).

Validity and Reliability: The PCL has a well-established reliability and validity assured by Weathers et al. ${ }^{[15]}$ It was revised by the same expert panel after translation.

\subsection{Piloting the instruments}

It was conducted to test feasibility and applicability of the instruments and also the maneuver of intervention. It was used to estimate the time needed for data collection. It was conducted on a sample of $10 \%$ of the total (4 women). They were excluded from the main sample. The results of piloting were used to finalize the instruments and schedule the field work time needed. Some changes were made to the instruments according to the findings of piloting.

\subsection{Ethical consideration}

This work was approved by the ethics committee of the Faculty of Nursing, Menoufia University. An official written approval was obtained from the Dean of Faculty of Nursing forwarded to the director of above mentioned settings to conduct the study. An oral consent was obtained from all studied women who met the inclusion criteria after informing them about the purpose and nature of the study. All collected data were strictly confidential and were used for scientific purposes only.

\subsection{Statistical data analysis}

The data collected were tabulated \& analyzed by SPSS (statistical package for the social science) version 20. Quantitative data were expressed as mean \& standard deviation $(\bar{X} \pm$ SD). Test of correlation was applied using Pearson test. Descriptive data were expressed as number and percentage (No $\& \%)$ and analyzed by applying chi-square test, $p$-value at .05 was used to determine significance regarding: $p$-value $>.05$ to be non-significant (NS); $p$-value $\leq .001$ to be high significant (HS).

\section{Results}

The sociodemographic characteristics of the studied participants are displayed in Table 1. The table shows that the mean age of participants was 27 years. As for education, the majority of participants were highly educated, employee, rural residents, living away from husband's family and having not enough monthly income.

Obstetric history of the participants is displayed in Table 2. As for the gravidity; about half of the sample was pregnant for the 5 th time. The majority of participants were not having living children due to recurrent abortion. More than half of women $(55 \%)$ of women experienced 4 to 5 abortions. The table also shows that the time between current pregnancy and previous abortion was from 3 to 4 months or more for $47.5 \%$ of participants.

Table 1. Socio-demographic characteristics of the studied participants

\begin{tabular}{lll}
\hline Variable & No (40) \\
\cline { 2 - 3 } & No & \% \\
\hline Age & $27.09 \pm 1.56$ & \\
Mean \pm SD & & \\
Level of education & - & - \\
Illiterate & 2 & 5 \\
Basic & 14 & 35 \\
Secondary & 24 & 60 \\
$\quad$ University & & \\
Women Occupation & 27 & 67.5 \\
Employee & 13 & 32.5 \\
House wife & & \\
Residence & 15 & 37.5 \\
Urban & 25 & 62.5 \\
Rural & & \\
Accommodation & 13 & 32.5 \\
With husband family & 27 & 67.5 \\
Alone & & \\
Monthly income & 17 & 57.5 \\
Enough & 23 & \\
Not-enough & & \\
\hline
\end{tabular}

Participants' feelings regarding current pregnancy are shown in Table 3. The table shows that the majority of women were ambivalent toward the suspected pregnancy. After pregnancy being confirmed, $17 \%$ of them were having negative feelings and $17 \%$ also were ambivalent.

Figure 1 displays the degree of symptoms severity at the first measurement (before the intervention). The figure displays that 11 women were having post traumatic stress disorder.

Figure 2 shows the degree of symptoms severity at the second measurement (after the intervention). The figure displays that 29 women were totally recovered.

Figure 3 shows that, there was a statistically significant difference between scores of PTS symptoms as perceived by the studied women before and after the intervention at $(p<$ .0001).

Table 4 shows that age, number of pervious abortion and time between current pregnancy and previous abortion were positively correlating with degree of post traumatic stress symptoms. This means that the symptoms severity are increasing with the increased woman's age, number of previous 
abortion and also with the fewer time elapsed between last abortion and current pregnancy.

Table 2. Obstetric history of the studied participants

\begin{tabular}{lll}
\hline \multirow{2}{*}{ Variable } & No (40) \\
\cline { 2 - 3 } & No & \% \\
\hline Gravidity & 6 & 15 \\
3 & 12 & 30 \\
4 & 21 & 52.5 \\
5 & 1 & 2.5 \\
6 & & \\
Having children & 11 & 27.5 \\
Yes & 29 & 72.5 \\
No & & \\
Gestational age & 32 & 80 \\
$1^{\text {st }}$ trimester & 8 & 20 \\
$2^{\text {nd }}$ trimester & & \\
Number of previous abortions & 18 & 45 \\
$2-3$ & 22 & 55 \\
$4-5$ & 28 & \\
Gestational age by weeks at previous abortion & 30 \\
$1-10$ weeks & 12 & 70 \\
$11-20$ weeks & 1 & 2.5 \\
Time between current pregnancy and previous abortion \\
1 month & 1 & 2.5 \\
2 months & 19 & 47.5 \\
3 months & 19 & 47.5 \\
4 months & \multicolumn{2}{l}{} \\
\hline
\end{tabular}

Table 4 also shows that there was a negative correlation between symptoms severity and education and having living children.

Table 3. Feelings regarding Current Pregnancy

\begin{tabular}{lcl}
\hline \multirow{2}{*}{ Variable } & \multicolumn{2}{c}{ No (40) } \\
\cline { 2 - 3 } & No & \% \\
\hline Feelings when current pregnancy & was suspected & \\
Positive & 5 & 12.5 \\
Negative & 17 & 42.5 \\
Ambivalent & 17 & 42.5 \\
Not specified & 1 & 2.5 \\
Feelings when current pregnancy was confirmed & \\
Positive & 2 & 5 \\
Negative & 12 & 30 \\
Ambivalent & 25 & 62.5 \\
Not specified & 1 & 2.5 \\
\hline
\end{tabular}

Table 5 shows that participants were having positive pregnancy outcomes. Table 5 supports the 2 nd hypothesis as it was found that after the intervention, women were having a more stable psychological status and in turn a positive pregnancy outcomes.

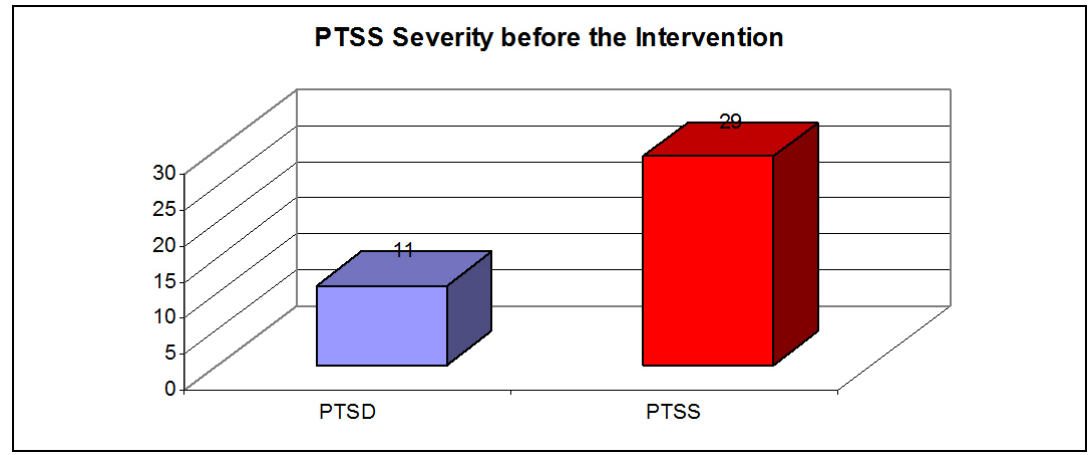

Figure 1. PTS symptoms severity at baseline (before intervention)

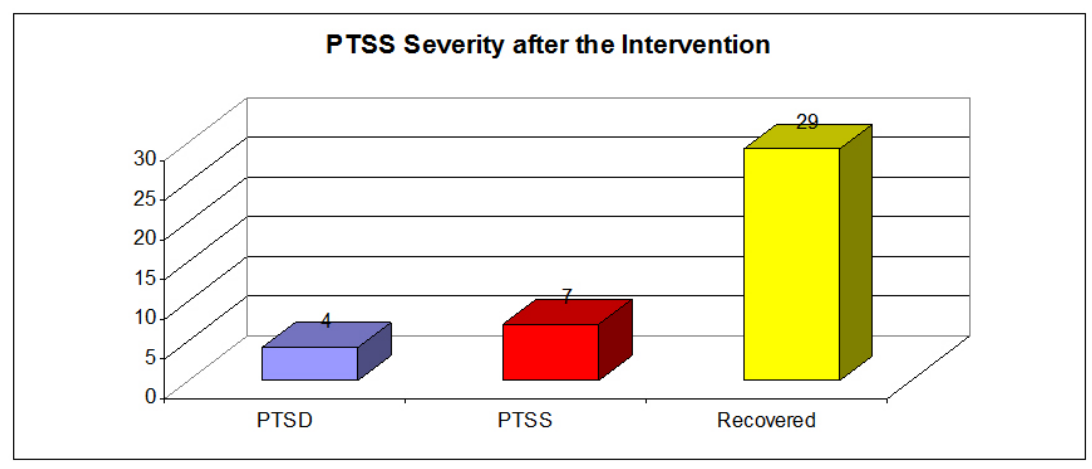

Figure 2. PTS symptoms severity after the 6th session of intervention 


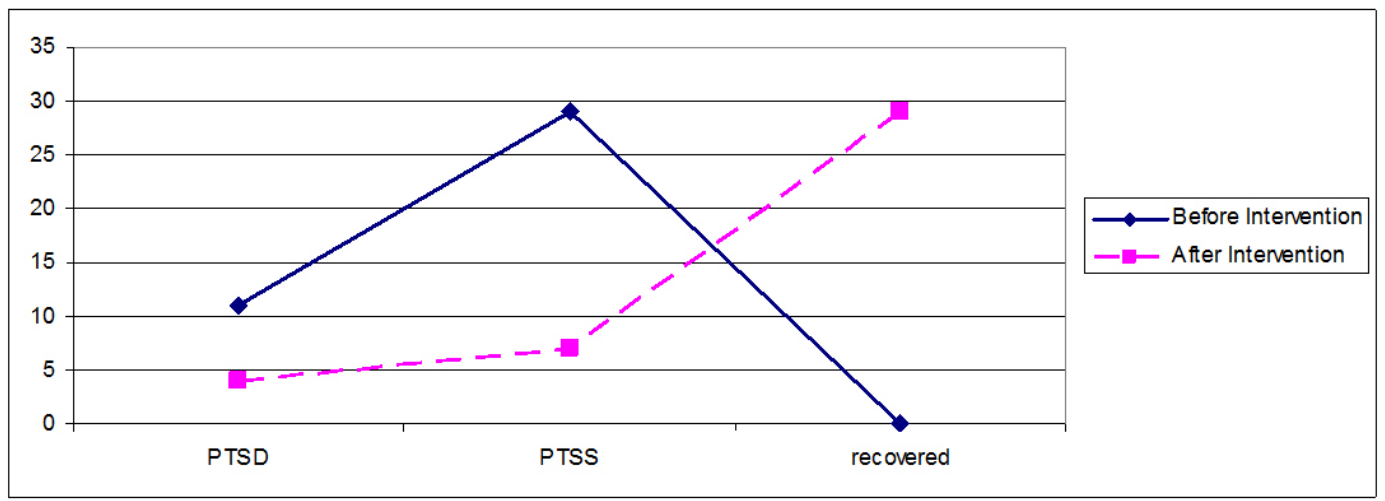

Figure 3. Level of PTS symptoms as Perceived by the Studied Women before and after the intervention Chi square test $=27.33, p$ value $<.0001$

Table 4. Correlation between Certain Variables and the Degree of Depressive Symptoms

\begin{tabular}{ll}
\hline \multirow{2}{*}{ Variable } & Degree of depressive symptoms \\
\cline { 2 - 2 } & Pearson correlation \\
\hline Age & 0.842 \\
Level of education & -0.854 \\
Having living children & -0.762 \\
Number of previous abortion & 0.773 \\
Time between current pregnancy and previous abortion & 0.801 \\
\hline
\end{tabular}

\section{Discussion}

The current study is one of the first which tried to pay attention to the critical point of having good psychological status during pregnancy to go through it safely and obtain positive outcomes.

The main intervention of the current study was a psychological intervention implemented in 6 consecutive sessions with a pre intervention measurement of symptoms severity followed by post sessions measurement and follow-up of pregnancy outcomes. By reviewing available systemic reviews summarizing similar studies it was found that; the main limitation of studies was that providing long-term psychological therapy can be expensive, and associated with high drop out level of studied women however it is considered the most exclusive nursing management strategy for PTSS. Few researches tried to address similar long term intervention like that of the current study. First one is a Swedish longitudinal abortion study, 12 of 58 women reported that they had been in a crisis during pregnancy following many abortions, while at the follow-up phase, only two women were still expressed feelings in relations to the abortion in terms of a crisis. ${ }^{[16]}$ Second study was conducted at the year 2011 by Beck et al. ${ }^{[17]}$ named "posttraumatic stress symptoms in recurrent abortion women: results from a two-stage U.S. national study" and generated similar improvement in symptoms severity after the long term management.
The mean age of the studied women was 27.09 years which is the mid fertility years. This finding is consistent with Slade et al. ${ }^{[10]}$ who studied women with a mean age of 28 years for predicting the psychological impact of miscarriage. Tennen et al. ${ }^{[18]}$ investigated self-blame among women with recurrent abortion and found that the mean age of their sample was 26 years.

The current findings revealed a strong positive correlation between the symptoms severity and age. In other terms; the symptoms were severe among older women than younger ones. The correlation is seen to be due to that women believe about having a limited fertility period. Women see repeated abortions as fertility time wasting and in turn became more depressed. Many studies supported this result. First; a study conducted in USA by Herz ${ }^{[19]}$ named "Perinatal loss in Psychological Aspects of Women's Health Care". Second; a study in Northern Ireland by Cecil and Leslie ${ }^{[20]}$ named "Early miscarriage and its psychological effects". Authors of both previously mentioned studies reported that female age was positively correlating with depressive symptoms severity.

As for the level of education; the findings showed that more than half of women were highly educated. Abiola et al. ${ }^{[21]}$ Reported a highly educated sample in a Nigerian study about the knowledge, prevalence and psychological effects of mis- 
carriage among women of reproductive age.

Table 5. Pregnancy Outcome among Studied Participants

\begin{tabular}{|c|c|c|}
\hline \multirow{2}{*}{ Variable } & \multicolumn{2}{|c|}{ No (40) } \\
\hline & No & $\%$ \\
\hline \multicolumn{3}{|c|}{ Reporting any pregnancy danger signs } \\
\hline Yes & & \\
\hline No & 0 & 0 \\
\hline \multicolumn{3}{|c|}{ Pregnancy complications } \\
\hline Anemia & 26 & 65 \\
\hline \multicolumn{3}{|l|}{ Mode of delivery } \\
\hline Normal & 19 & 47.5 \\
\hline Sc & 21 & 52.5 \\
\hline \multicolumn{3}{|l|}{ Obstructed labor } \\
\hline Yes & 3 & 7.5 \\
\hline No & 37 & 92.5 \\
\hline \multicolumn{3}{|l|}{ Laceration } \\
\hline Yes & 1 & 2.5 \\
\hline No & 39 & 97.5 \\
\hline \multicolumn{3}{|l|}{ Extended episiotomy } \\
\hline Yes & 4 & 10 \\
\hline No & 36 & 90 \\
\hline \multicolumn{3}{|c|}{ Postpartum complications } \\
\hline Yes & 7 & 17.5 \\
\hline No & 33 & 82.5 \\
\hline \multicolumn{3}{|l|}{ Fetal distress } \\
\hline Yes & 0 & \\
\hline No & 0 & \\
\hline \multicolumn{3}{|l|}{ Apgar score at 1} \\
\hline $0-4$ & 13 & 32.5 \\
\hline $4-6$ & 13 & 32.5 \\
\hline $7-10$ & 14 & 35 \\
\hline \multicolumn{3}{|l|}{ Apgar score at 5} \\
\hline $0-4$ & 3 & 7.5 \\
\hline $4-6$ & 13 & 32.5 \\
\hline $7-10$ & 24 & 60 \\
\hline \multicolumn{3}{|l|}{ Neonatal maturity } \\
\hline Full term and alive & 39 & 97.5 \\
\hline Premature and alive & 1 & 2.5 \\
\hline \multicolumn{3}{|l|}{ Birth weigh } \\
\hline Low birth weight & 2 & 5 \\
\hline Normal birth weight & 38 & 95 \\
\hline \multicolumn{3}{|l|}{ Birth injuries } \\
\hline Yes & 0 & 0 \\
\hline No & 0 & 0 \\
\hline \multicolumn{3}{|l|}{ Need for resuscitation } \\
\hline Yes & 5 & 12.5 \\
\hline No & 35 & 87.5 \\
\hline \multicolumn{3}{|c|}{ Admission to incubator } \\
\hline Yes & 7 & 17.5 \\
\hline No & 33 & 82.5 \\
\hline \multicolumn{3}{|l|}{ Admission to NICU } \\
\hline Yes & 2 & 5 \\
\hline No & 38 & 95 \\
\hline \multicolumn{3}{|l|}{ Congenital anomalies } \\
\hline Cleft lip & 1 & 2.5 \\
\hline No & 39 & 97.5 \\
\hline
\end{tabular}

The current findings showed a negative correlation between educational level and symptoms severity. The symptoms were less severe among highly educated women. This can be rationalized by the idea that educated women were more aware of their health problem and can easily cope with. Lok et al. ${ }^{[22]}$ published a study named "A 1-year longitudinal study of psychological morbidity after repeated miscarriage". The published data showed a similar negative correlation between educational level and symptoms severity. In contrast, in a study named "stress and anxiety levels following first-trimester miscarriage", Cheung et al. ${ }^{[23]}$ reported no correlation between the aforementioned factors. The contradiction in results may be due to the difference in sample nature. Cheung et al. ${ }^{[23]}$ conducted the study among women conceived with assisted reproduction and their symptoms severity was highly affected with their bad psychological status regardless of their educational level.

The current study showed that only one fourth of the sample was having living children. Scotchie and Fritz ${ }^{[24]}$ studied the effect of early pregnancy loss published similar percentage as some of their studied sample was having living children and failed to go through pregnancy after that.

Having living children or even child was playing a role in decreasing symptoms severity. It was noticed that having living children was negatively correlating with symptoms severity. This is because such women see having living child as a measure to relieve tension and depression compared to being nullipara. Similar correlation was reported by Nikcevic ${ }^{[7]}$ in a study of psychological outcomes following abortions and provision of follow-up care. Meanwhile, Cuisinier et al. ${ }^{[25]}$ reported contradicted results in a study of the course of grief and some determining factors during pregnancy following miscarriage. The difference in findings may be due to that Cuisinier et al. ${ }^{[25]}$ studied women had just recovered from secondary infertility and were in very bad psychological status not affected even by the presence of living children.

Half of the studied women experienced four to five previous abortions times. Similar findings were published by Séjourné et al. ${ }^{[13]}$ in a study conducted in Pakistan to detect prevalence of PTSS following recurrent abortion.

The number of previous abortion was positively correlating with symptoms severity. Increased times of previous abortions was associated with increased symptoms severity. The rationale is that the experience of recurrent abortion was a traumatic event and resulted in PTSS. Repeating the traumatic experience directly increases symptoms severity. By reviewing available body of knowledge; it was found 
that Geller et al. ${ }^{[2]}$ made a systemic review of nearly all researches investigated PTSS following abortion in a giant study named "PTSS following recurrent miscarriage during the subsequent pregnancy: A review of the literature and future directions". This review showed that all studies revealed the same finding regarding this point.

Women were purposively selected with 1 to 4 months difference between pregnancy and last abortion. Nearly all were experiencing current pregnancy three to four months after the last abortion time. Women tried to take a rest period to be able to go through pregnancy safely. The finding is in accordance with Major et al. ${ }^{[26]}$ who studied repeated abortion and mental health among similar sample attitude. Women in the aforementioned study preferred to take a rest period between abortion and subsequent pregnancy.

The time elapsed after last abortion was positively correlating with the experience of PTSS. This is because the PTSS slightly decrease in severity with new pregnancy experience and the delay in pregnancy occurrence increase the symptoms severity. Charles et al. ${ }^{[3]}$ published a systematic review in 2015 about repeated abortion and long-term mental health outcomes and reported similar findings. Nearly all studies included in that review indicated a similar positive correlation.

As for feelings regarding the current pregnancy; the findings showed that the majority of participants were feeling negative and ambivalent toward the diagnosis of being pregnant. This is why they predict the same end to their pregnancy as the previous times which was abortion. Breslau et al. ${ }^{[27]}$ surveyed the psychiatric sequel of posttraumatic stress disorder in recurrent abortive women and reported that women had ambivalent feelings toward pregnancy. Major et al. ${ }^{[28]}$ studied psychological responses of women after repeated first-trimester abortion and reported a contradicted result. In Major et al.' ${ }^{[28]}$ study, women were feeling positive and delight toward pregnancy because they were receiving a psychotherapy course 2 months prior to pregnancy.

While measuring PTSS severity for the first time and as a baseline data; the results showed that more than half of women were having severe PTSS. After the 6th session of the intervention; nearly half of women were recovered. The decline in symptoms severity is rationalized by the effect of the psycho-educational program sessions. A study by Faramarzi and Pasha ${ }^{[29]}$ about the role of psycho-educational program in managing stress during pregnancy after repeated abortion showed similar finding. Faramarzi and Pasha's ${ }^{[29]}$ study reveled an improvement in PTSS among studied women after receiving similar psycho-educational program. On the contrary, Steinberg and Russo ${ }^{[30]}$ studied abortion, PTSD and PTSS reported limited improvement in severity. The con- tradiction is seen to be due to high drop out level of sample compared to the current study.

The current findings showed that studied women had positive pregnancy (maternal and neonatal) outcomes without any reported major complications. This finding highlights how important it is that with good psychological status; woman can go through pregnancy safely and vise versa. Importantly, this finding is in line with a number of studies suggesting the importance of psychological intervention to have positive pregnancy outcomes. For example that done by Robinson et al. ${ }^{[31]}$ about pregnancy outcomes of women with "PTSS". One year later, Coleman ${ }^{[4]}$ published a survey named "abortion and mental health: quantitative synthesis and analysis of published researches from 1995-2013" and highlighted that three studies reported positive pregnancy outcomes after receiving psychological intervention for PTSS.

Few observational studies with cohort designs and large sample sizes failed to show any association between psychological intervention and pregnancy outcomes. The most obvious is that by Cougle et al., ${ }^{[32]}$ Coleman $^{[4]}$ which evaluated effect of psychological sessions on depression associated with recurrent abortion. Researchers of this study reported a high drop-out level of studied women and thus a low generalizability of results.

\section{Conclusion}

Women experiencing prenatal loss should have a customized management plan that may include education, resources, and the opportunity to discuss their loss. Effective management for PTSS should focus on psychological intervention as the lack of such important intervention is considered a risk factor for developing post traumatic stress disorder following reproductive trauma.

Regarding the effect of psychological intervention on posttraumatic stress symptoms and pregnancy outcomes; the findings of the currents study revealed that the intervention was effective in relieving PTSS and improved the psychological wellbeing of women thus leads to positive pregnancy outcomes.

Based on the current study findings, both study hypotheses were accepted as it was clearly noticed that: The psychological intervention succeeded in decreasing post-traumatic stress symptoms severity among women with recurrent abortion. The psychological intervention improved pregnancy outcomes among women with recurrent abortion.

\subsection{Recommendations}

- Manage women with recurrent abortion within the purview of holistic nursing practice which address the

ISSN 1925-4040 E-ISSN 1925-4059 
bio-psychosocial responses of abortion.

- Adopt intervention strategies that focus on resolving the psychological consequences of recurrent abortion and its impact on women subsequent pregnancy outcomes.

- Integrate the psychological assessment in the antenatal care to identify women at risk for psychological problems.

\subsection{Study limitations}

This is a non-randomized study with purposive sample. This limits the inferences drawn from practice.

\section{CONFLicts OF INTEREST Disclosure}

The authors declare that there is no conflict of interest.

\section{REFERENCES}

[1] Michale O. Recurrent abortion and associated psychological symptoms. Int J Gynecol Obstetr. 2016; 18: 202-15.

[2] Geller P, Kerns D, Klier C. PTSS following recurrent miscarriage during the subsequent pregnancy: A review of the literature and future directions. J Psychosom Res. 2017; 56: 35-45. https://doi.org/10.1016/S0022-3999(03)00042-4

[3] Charles V, Polis C, Sridhara S, et al. Repeated abortion and longterm mental health outcomes: a systematic review of the evidence. Contraception. 2015; 78: 436-450. PMid:19014789 https : //doi.org/10.1016/j.contraception.2008.07.005

[4] Coleman P. Abortion and mental health: quantitative synthesis and analysis of research. Br J Psychiatry. 2013; 199: 180-186.

[5] Swanson V, Akker O, Purewal S. Posttraumatic stress and posttraumatic stress disorder after termination of pregnancy and reproductive loss: a systematic review. J Psychosom Obstet Gynecol. 2015; 12: 165-173.

[6] American Psychological Association. Report of the Task Force on Mental Health and Abortion. Washington, DC: American Psychological Association 2008. Available from: http://www .apa.org/pi /women/programs/abortion/mental-health.pdf

[7] Nikcevic G. Posttraumatic stress among women after induced abortion: a Swedish multi-centre cohort study. BMC Women's Health. 2013; 13: 52. PMid:24364878 https://doi.org/10.1186/1472 $-6874-13-52$

[8] Roswell J, Kilby K, Orford A. How women who have experienced one or more miscarriages manage their feelings and emotions when they become pregnant again: a qualitative interview study. Scandinavian J Caring Sci. 2011; 26(2): 262-270. PMid:21967678

[9] Adolfsson B, Larsson J. Post-traumatic stress disorder in women who have undergone obstetric and/or gynaecological procedures. J Reprod Infant Psyc. 2016; 11: 221-228.

[10] Lee S, Lygo JC, Atrash H. Psychological impact of miscarriage: United States. Am J Public Health. 2016; 93: 631-634.

[11] Neugebauer D, Macha A, Stankowska I, et al. What should women do to overcome PTSD. Woman Psychology. 2017; 8(1): 41-6.

[12] van den Hout E, Arntz M, Kutteh W. Evaluation and management of recurrent early pregnancy loss. Clin Obstet Gynecol. 2016; 1: 132-145.

[13] Séjourné A, Callahan U, Chabrol L. Post Traumatic stress during pregnancy. Probl Psychiat. 2011; 12(4): 420-7.

[14] Polit F, Beck C. Essential of nursing research and methods; Research design. 7th ed., Lippincott William \& Wilkins, New York: 2001; 39(42): 165-230.

[15] Weathers F, Litz B, Herman D, et al. The PTSD checklist (PCL): reliability, validity, and diagnostic utility. 1993.
[16] The Swedish National Board of Health and Welfare-Health and Medical Care: recurrent abortions. Social styrelsen/Official Statistics of Sweden. 2010. Available from: http://www. socialstyrelsen.se/lists/artikelkatalog/ attachments $/ 18330 / 2011-6-1 . p d f$

[17] Beck C, Gable R, Sakala C, et al. Posttraumatic stress symptoms in recurrent abortion women: results from a two-stage U.S. national study. Birth. 2011; 38: 216-227.

[18] Tennen H, Affleck G, Gershman K. Self-blame among women with recurrent abortion: the role of self-protective motives. J Pers Soc Psychol. 2016; 50: 690-696. https://doi.org/10.1037/0022 $-3514.50 .4 .690$

[19] Herz E. Perinatal loss in Psychological Aspects of Women's Health Care, edited by Stewart D, Stot J: American Psychiatric Press; 2013. 140-160 p.

[20] Cecil R, Leslie J. Early miscarriage and its psychological effects: preliminary results from a study in Northern Ireland. Journal of Reproductive and Infant Psychology. 2016; 11: 89-95.

[21] Abiola A, Ajayi A, Umeh C, et al. Knowledge, prevalence and psychological effect of miscarriage among women of reproductive age group attending obstetrics and gynaecology clinics of Lagos University Teaching Hospital. Niger Postgrad Med J. 2013; 20(4): 319-324.

[22] Lok I, Yip A, Lee D, et al. A 1-year longitudinal study of psychological morbidity after repeated miscarriage. Fertil Steril. 2012; 93(6): 1966-1975.

[23] Cheung C, Chan C, Ngo E. Stress and anxiety levels following first-trimester miscarriage among women conceived with assisted reproduction. BJOG. 2013; 120(9): 1090-1097. PMid:23631687 https ://doi.org/10.1111/1471-0528.12251

[24] Scotchie J, Fritz M. Effect of early pregnancy loss. Postgrad Obstet Gynecol. 2015; 26(9): 1-7.

[25] Cuisinier M, Janssen H, De Graauw C, et al. Pregnancy following miscarriage: Course of grief and some determining factors. J Psychosom Obstet Gynecol. 2016; 17: 168-174. https ://doi .org/10.3 109/01674829609025678

[26] Major B, Appelbaum M, Beckman L, et al. Repeated abortion and mental health: Evaluating the evidence. Am Psychol. 2014; 64: 86390. PMid:19968372 https://doi .org/10.1037/a0017497

[27] Breslau N, Davis G, Peterson E, et al. Psychiatric sequelae of posttraumatic stress disorder in recurrent abortive women. Arch Gen Psychiatry. 2014; 54: 81-87. https://doi.org/10.1001/arch psyc.1997.01830130087016

[28] Major B, Cozzarelli C, Cooper M, et al. Psychological responses of women after repeated first-trimester abortion. Arch Gen Psychiatry. 2013; 57: 777-784. https://doi.org/10.1001/archpsyc. 57. 8.777

Published by Sciedu Press 
[29] Faramarzi M, Pasha H. The role of psycho-educational program in managing stress during pregnancy after repeated abortion. J Babol Univ Med Sci. 2015; 17(11): 52-60.

[30] Steinberg J, Russo N. Abortion and PTSD and PTSS: what's the relationship? Soc Sci Med. 2013; 67: 238-252.
[31] Robinson G, Stotland N, Russo N, et al. What are the pregnancy outcomes of women with "PTSS"? Critiquing the evidence. Harv Rev Psychiatry. 2012; 17: 268-290.

[32] Cougle J, Reardon D, Coleman P. Depression associated with recurrent abortion. Med Sci Monitor. 2011; 9: 105-12. 